\title{
Fermi Arcs in Tilted Weyl Semimetals: Classification, Evolution and Transport Properties
}

\author{
Xiao-Ming Zhao, ${ }^{1}$ Xiao Kong, ${ }^{1}$ Cui-Xian Guo, ${ }^{1}$ Ya-Jie Wu, ${ }^{2}$ and Su-Peng Kou ${ }^{1}, *$ \\ ${ }^{1}$ Center for Advanced Quantum Studies, Department of Physics, \\ Beijing Normal University, Beijing 100875, China \\ ${ }^{2}$ School of Science, Xi'an Technological University, Xi'an 710021, China
}

\begin{abstract}
The Weyl semimetal is a new quantum state of topological semimetal, of which topological surface states - the Fermi arcs exist. In this paper, the Fermi arcs in Weyl semimetals are classified into two classes - class-1 and class-2. Based on a tight-binding model, the evolution and transport properties of class-1/2 Fermi arcs are studied via the tilting strength of the bulk Weyl cones. The (residual) anomalous Hall conductivity of topological surface states is a physical consequence of class-1 Fermi arc and thus class-1 Fermi arc becomes a nontrivial topological property for hybrid or type-II Weyl semimetal. Therefore, this work provides an intuitive method to learn topological properties of Weyl semimetal.
\end{abstract}

\section{INTRODUCTION}

Topological materials, including topological superconductors, topological superfluids, topological insulators and topological semimetals become more and more important in condensed matter physics. The Weyl semimetal (WSM) is a new type of topological semimetals [1-10]: a three dimensional (3D) version of the graphene with several bulk gapless points in the reciprocal space, i.e., Weyl nodes. Each Weyl node can be regarded as a monopole in the reciprocal space, carrying a topological charge of \pm 1 corresponding to the left-hand or right-hand chirality. The Weyl nodes are separated in momentum space and exist in pairs which are connected only through topological surface states - the Fermi arcs (FAs). The recent progress in identified WSM materials [11-15] has driven a flurry of exciting researches to probe the various fascinating phenomena connected to Weyl fermions, such as the chiral anomaly and the chiral magnetic effect [16-19]. The WSMs have been discovered in the TaAs family where it features multiple Fermi arcs arising from topological surface states[20,21], and exhibits novel quantum phenomena, e.g., chiral anomaly induced negative magnetoresistance $[22,23]$ and possibly emergent supersymmetry [24].

Recently, it was proposed theoretically and experimentally that a new type (type-II) of WSM [25-27] can emerge with topologically-protected touching between electron and hole pockets. The topological surface states were confirmed by directly observing the surface states using bulk and surface-sensitive angle-resolved photoemission spectroscopy (ARPES). Besides, the third type of WSM is the hybrid one (or type-1.5) in which one Weyl node belongs to type-I whereas its chiral partner belongs to the type-II. The type-II WSM has been proposed to exist in layered transition metal dichalcogenides, such as $\mathrm{W}_{x} \mathrm{Mo}_{1-x} \mathrm{Te}_{2}[28-30]$, and the tight-binding models for

\footnotetext{
${ }^{*}$ Corresponding author; Electronic address: spkou@bnu.edu.cn
}

the hybrid WSM have been constructed in possible materials and optical lattices [31, 32].

It is known that for usual quantum Hall systems, the current-carrying chiral edge states are responsible for the integer quantized Hall conductance which can be measured by the transport experiments[33, 34]. The anomalous Hall conductivity (AHC) for a type-I WSM with two Weyl nodes is proportional to the distance of the Weyl points[5]. When tilting the energy dispersion of type-I WSM enough along a certain direction we get a hybrid or type-II WSM, and the Weyl cones could even be tipped over so that the Fermi surface transforms from a point to a line or a surface. Both the surface states and bulk states have contribution to the Hall conductance. For type-II WSM the Hall conductivity is not universal and can change sign as a function of the parameters quantifying the tilting strength in Ref.[35], where they only considered the low energy approximation of the bulk states. For type-II and hybrid type WSMs, the surface states make significant contribution to the Hall conductance. However, it is not known how the AHC is related to the surface states for type-II and hybrid type WSMs. The main purpose of the paper is to make it clear.

This paper is organized as follows. In Sec.II, we define the two classes of FAs generally and induce a dimensionless parameter - residual class-1 Fermi arc - to describe the residual effects of the Fermi arc. And then based on a tight-binding model, the evolution of residual Fermi arc with the tilting strength of the Weyl cones is shown in Sec.III. Furthermore, Sec.IV shows the effects of residual Fermi arcs on the anomalous Hall conductivity and the restrict relationship between them is figured out. Finally, we conclude our research in Sec.V.

\section{CLASSIFICATION OF THE FERMI ARCS}

A low-energy Hamiltonian of a WSM is written as

$$
H_{\mathrm{W}}(k)=\sum_{i}\left(v_{i} \sigma_{i}+C_{i}\right) k_{i}
$$




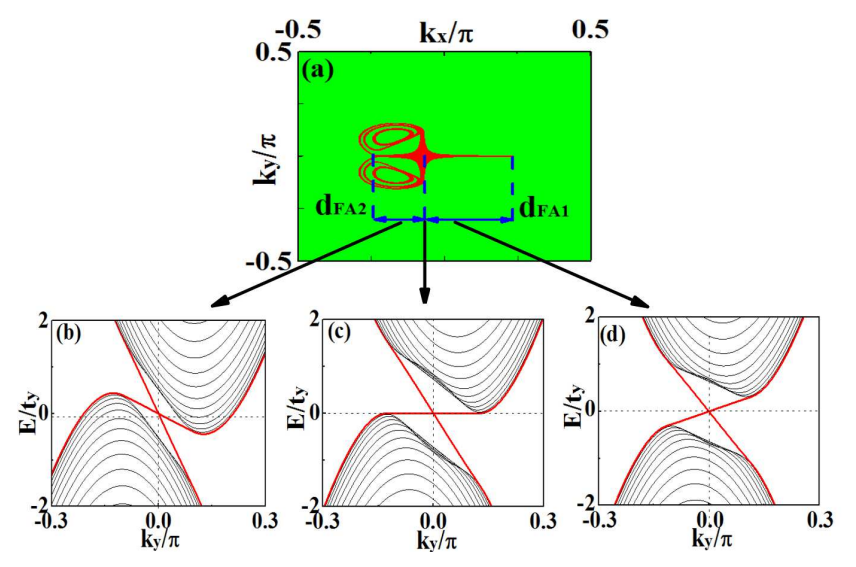

FIG. 1: (Coloronline) Coexistence of class-1 and class-2 Fermi arcs in hybrid type WSM. (a) The two classes of Fermi arcs locate at $k_{y}=0$, which are denoted by blue arrowed lines. The red arcs denote the shape of electron pockets and hole pockets. (b-d) Energy dispersion of the surface states (denoted by red line) for two classes of Fermi arcs.

where $\sigma_{i}(i=x, y, z)$ is the $2 \times 2$ Pauli matric, $v_{i}$ is the Fermi velocity, $C_{i}$ denotes the tilting strength of the linear energy dispersion along $k_{i}$ which breaks Lorentz invariance. The Weyl nodes are characterized by a topological Chern number $n_{W}=\operatorname{sgn}\left[\prod_{i} v_{i}\right]= \pm 1$. The Weyl nodes have been classified into type-I for the case of $\left|C_{i} / v_{i}\right|<1(i=x, y, z)$, and type-II for the case of $\left|C_{i} / v_{i}\right|>1(i=x, y, z)$.

In a WSM, a paired Weyl nodes with opposite chiralities are connected through the Fermi arc. Generally, when we take open boundary condition (OBC) along one direction (here take z-direction as an example) the surface states cross Fermi surface $E_{f}$ somewhere marked by $k_{\lambda}=\left(k_{\lambda, x}, k_{\lambda, y}\right)$ and eventually the cross points constitute the Fermi arc. The surface states near a certain point $k_{\lambda}$ can also be described by a linear model as

$$
H_{\mathrm{S}}\left(k_{\lambda}\right)=\left(v_{s} \sigma_{z}+C_{\lambda, \perp}\right) \mathbf{k}_{\lambda, \perp}
$$

where $\mathbf{k}_{\lambda, \perp}$ is the momentum perpendicular to Fermi arc, $C_{\lambda, \perp}$ represents the tilting strength along the $\mathbf{k}_{\lambda, \perp}$ direction. Similar to the classification of Weyl nodes which is based on the tilting strength of the bulk energy dispersion, we classify the Fermi arcs into two classes that are denoted by

$$
\begin{aligned}
& \text { Class-1 (FA1): }\left|C_{\lambda, \perp}\right| / v_{s}<1, \\
& \text { Class-2 (FA2): }\left|C_{\lambda, \perp}\right| / v_{s}>1 .
\end{aligned}
$$

Because FA1 (FA2) exists near type-I (type-II) Weyl node, in the hybrid WSM, the FA1 and FA2 may coexist. The schematic diagram of the two classes of Fermi arcs is shown in Fig.1. When the type-I WSM changes into hybrid or type-II, the region of FA1 shrinks and the region of FA2 enlarges. To characterize the evolution of two classes of Fermi arcs, we introduce a dimensionless
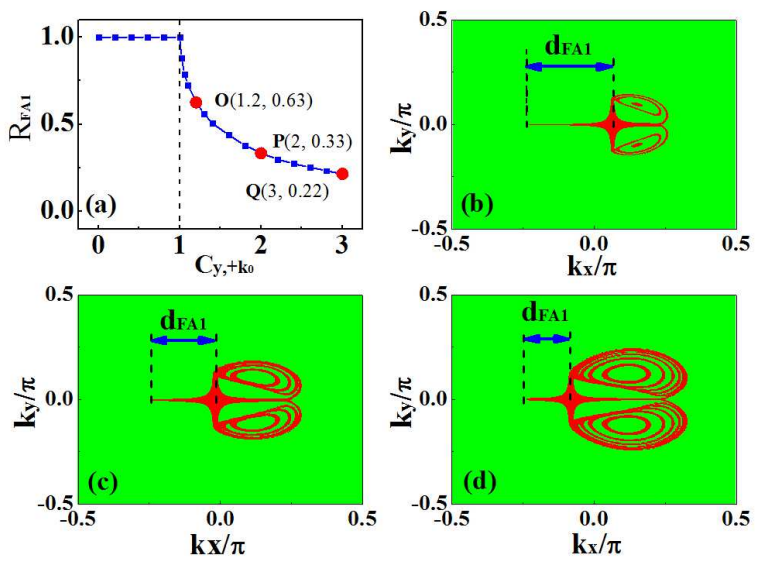

FIG. 2: (Coloronline) The evolution and appearance of the residual class-1 Fermi arc via tilting strength for case-1. Case-1: WSM changes from type-I to hybrid type. (a) The evolution of residual ratio of class-1 Fermi arc $R_{\mathrm{FA} 1}$. (b-d) The appearance of residual class-1 Fermi arc corresponding to the situation at $\mathbf{O}, \mathbf{P}, \mathbf{Q}$ respectively, which are marked by red solid dots in panel (a) and the tilting strength are $C_{y,+k_{0}}=1.2,2,3$. The residual FA1 locates at left part of Fermi arc

parameter, i.e., residual ratio of class-1 Fermi arc

$$
R_{\mathrm{FA} 1}=\frac{d_{\mathrm{FA} 1}}{d_{0, \mathrm{FA}}}=\frac{\mathbf{d}_{\mathbf{F A} 1} \cdot \mathbf{e}_{\mathbf{l}}}{\mathbf{d}_{\mathbf{0 , F A}} \cdot \mathbf{e}_{\mathbf{l}}}
$$

where $\mathbf{d}_{\mathbf{F A 1}}\left(\mathbf{d}_{\mathbf{0}, \mathbf{F A}}\right)$ represents the length-vector of FA1 (FA) from positive chiral Weyl node to negative chiral Weyl node, and $d_{\mathrm{FA} 1}\left(d_{0, \mathrm{FA}}\right)$ is the projection of $\mathbf{d}_{\mathbf{F A} \mathbf{1}}$ ( $\left.\mathbf{d}_{\mathbf{0}, \mathbf{F A}}\right)$ on the direction $\mathbf{e}_{\mathbf{l}}$ in momentum space. Remember that the length $d_{0, \mathrm{FA}}$ of the FA is constant due to topological protection of the Weyl nodes.

\section{EVOLUTION OF TWO CLASSES OF FERMI ARCS IN TILTED WEYL SEMIMETALS}

To illustrate the effects of the two classes of Fermi arcs, and for simplicity but without loss of generality, we consider a tight-binding model on the cubic lattice, of which the Hamiltonian in momentum space is given by

$$
\begin{aligned}
H(k) & =\left[2 t_{x}\left(\cos k_{x}-\cos k_{0}\right)+m\left(2-\cos k_{y}-\cos k_{z}\right)\right] \sigma_{x} \\
& +2 t_{y} \sin k_{y} \sigma_{y}+A_{1} \sin \left(k_{x}+k_{0}\right) \sin k_{y} \sigma_{0} \\
& +2 t_{z} \sin k_{z} \sigma_{z}+A_{2} \sin \left(k_{x}-k_{0}\right) \sin k_{y} \sigma_{0}
\end{aligned}
$$

where $t_{x}\left(t_{y}, t_{z}\right)$ corresponds to the nearest neighbor hopping parameter along $x(y, z)$-direction of the lattice. When $m>\left|t_{x}\left(1+\cos k_{0}\right)\right|$, there are two Weyl points located at $\mathbf{k}=\left( \pm k_{0}, 0,0\right) . A_{1}\left(A_{2}\right)$ leads to the tilting effect of the Weyl cone at $k_{x}=k_{0}\left(-k_{0}\right)$ along $y$-direction. In this paper, we set $k_{0}=\frac{\pi}{4},-t_{x} \sin k_{0}=t_{y}=t_{z}=1$. Corresponding to the linear-part of the Weyl node described in Eq.1, we define the tilting strength near $k_{x}=$ 

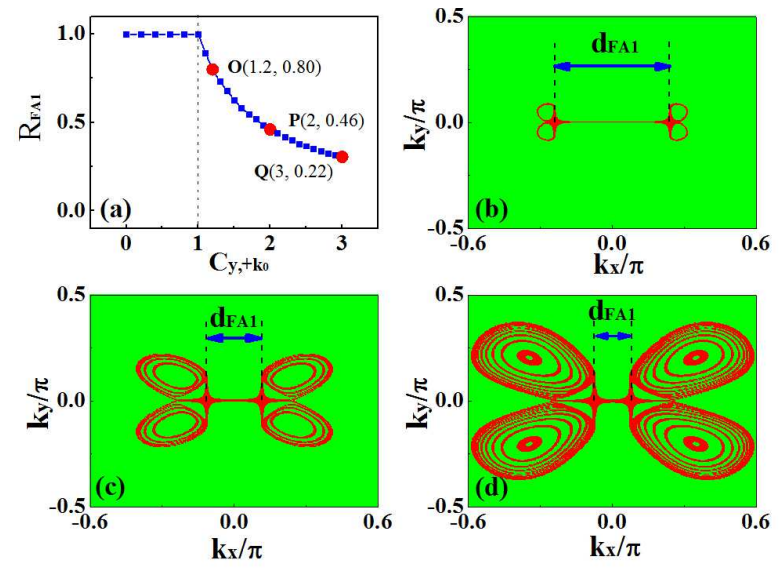

FIG. 3: (Coloronline) The evolution and appearance of the residual class-1 Fermi arc via tilting strength for case-2. case-2: WSM changes from type-I to type-II (node tilting to opposite directions along $k_{y}$ direction). (a-d) Same as panel (a-d) in Fig.2. The tilting strength are $C_{y,+k_{0}}=$ $1.2,2,3$. The residual FA1 locates at middle part of Fermi arc.

$$
\begin{aligned}
& +k_{0},-k_{0} \text { as } \\
& \qquad\left|C_{y,+k_{0}}\right|=-\frac{A_{1} \cos \left(k_{0}\right)}{t_{x}}, \quad\left|C_{y,-k_{0}}\right|=\frac{A_{2} \cos \left(k_{0}\right)}{t_{x}}
\end{aligned}
$$

respectively. The sign of $C_{y,+k_{0}} / C_{y,-k_{0}}$ indicates tilting direction parallel $(+)$ or antiparallel $(-)$ to $y$-direction. So when $\left|C_{y,+k_{0}}\right|<1$ and $\left|C_{y,-k_{0}}\right|<1$ the WSM is type-I, when $\left|C_{y,+k_{0}}\right|>1$ and $\left|C_{y,-k_{0}}\right|>1$ it is type-II, otherwise it is hybrid type.

Based on above model, we study the evolution of two classes of Fermi arcs in tilted Weyl semimetals. We choose the $\mathrm{OBC}$ along $z$-direction and periodic boundary condition (PBC) along $x / y$-direction. So the momentumdependence of the density of states (DOS) is given by

$$
\rho\left(k_{x}, k_{y}\right)=\sum_{i_{z}=1}^{N_{z}}-\frac{1}{\pi} \operatorname{Tr}\left[\operatorname{Im} \mathbf{G}\left(k_{x}, k_{y}, i_{z}\right)\right],
$$

here $\mathbf{G}\left(k_{x}, k_{y}, i_{z}\right)=\left[\mathbf{Z}-\mathbf{H}\left(k_{x}, k_{y}, i_{z}\right)\right]^{-1}$ is the Green's function of the $i_{z}$-th lattice layer along $z$-direction, where $\mathbf{Z}=(E+i \eta) \mathbf{I}$ is the complex energy, $E$ is energy level, $\eta$ is a infinite small value and $\mathbf{I}$ represent the unit matrix.

Fig.2-4(a) shows the evolution of residual ratio of class1 Fermi arc $R_{\mathrm{FA} 1}$ during the evolution of WSM types by tilting the two nodes toward $y$-direction. We show the results for three different cases: case-1 (Fig.2): WSM changes from type-I to hybrid type by tilting the single node at $k_{x}=+k_{0}\left(C_{y,+k_{0}}>0, C_{y,-k_{0}}=0\right)$; case-2 (Fig.3): WSM changes from type-I to typeII by tilting the two nodes along opposite direction $\left(C_{y,+k_{0}}=C_{y,-k_{0}}\right)$; case-3 (Fig.4): WSM changes from type-I to type-II by tilting the two nodes along same direction $\left(C_{y,+k_{0}}=-C_{y,-k_{0}}\right)$. While panels (b-d) show the features of the distribution of states on Fermi surface.
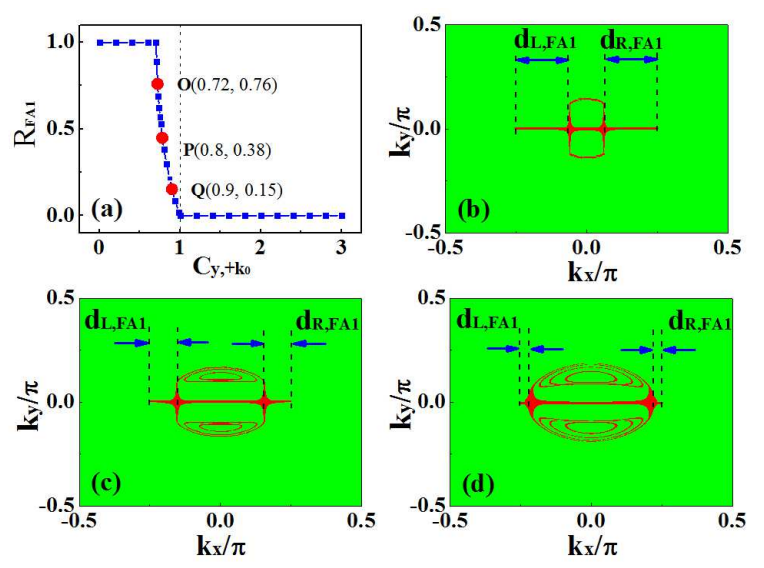

FIG. 4: (Coloronline) The evolution and appearance of the residual class-1 Fermi arc via tilting strength for case-3. Case-3: WSM changes from type-I to typeII (node tilting to same directions along $k_{y}$ direction).(ad) Same as panel (a-d) in Fig.2. The tilting strength are $C_{y,+k_{0}}=0.72,0.8,0.9$. The residual FA1 have two separated pieces located at both left and right ends.

The residual Fermi arcs in three cases are marked by red $\operatorname{dots} \mathbf{O}, \mathbf{P}, \mathbf{Q}$ in panel (a) respectively.

On the one hand, for the type-I WSM, the tilting strength is smaller than the critical value, i.e., $\left|C_{y,+k_{0}}\right|<$ 1 and $\left|C_{y,-k_{0}}\right|<1$, we have $d_{\mathrm{FA} 1}=d_{0, \mathrm{FA} 1} \equiv 2 k_{0}$. On the other hand, for the type-II or hybrid type WSM, the tilting strength is larger than the critical value, i.e., $\left|C_{y,+k_{0}}\right|>1$ or $\left|C_{y,-k_{0}}\right|>1$, several pairs of electronpockets and hole-pockets emerge on the Fermi surface which are induced by bulk states. For this case, FA1 and FA2 coexist. The residual FA1 locates at left part of Fermi arc in case-1 and middle part in case-2. In case3 the residual FA1 have two separated pieces located at both ends, of which the total length of the residual FA1 is written as $d_{\mathrm{FA} 1}=d_{\mathrm{L}, \mathrm{FA} 1}+d_{\mathrm{R}, \mathrm{FA} 1}$ (see Fig.4).

\section{ANOMALOUS HALL CONDUCTIVITY INDUCED BY RESIDUAL CLASS-1 FERMI ARC}

Anomalous Hall effect is a topological property in WSMs, and changing of topological surface states changes transport properties in WSMs, especially for AHC. In this part, we explore the relationship between the residual FA1 and AHC for different types of WSMs.

For a WSM, the 3D sample can be divided into 2D slices along a given direction[1]. In the WSM with two Weyl nodes mentioned above, the 2D slices for $k_{x} \in$ $\left(-k_{0}, k_{0}\right)$ can be regarded as a $2 \mathrm{D}$ topological insulator and the FA is the set of edge states corresponding to the $2 \mathrm{D}$ integer quantum Hall states. So the AHC of the WSM is proportional to the distance of the Weyl nodes in the momentum space, i.e., $\sigma_{0, y z}=e^{2} k_{0} / \pi h$.

Then we discuss the transport properties for the hybrid/type-II WSM. To characterize the topological 


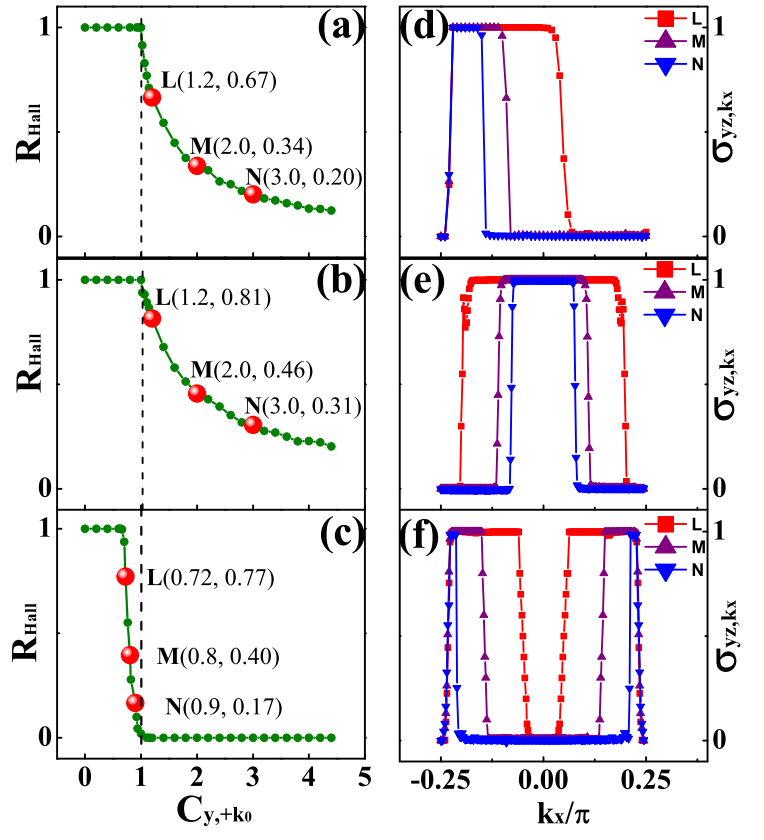

FIG. 5: (Coloronline) The evolution and momentumdependence of anomalous Hall conductivity. (a) The evolution of residual anomalous Hall conductivity $R_{\text {Hall }}$ for the situation of case-1. (d) The anomalous Hall conductivity structure in $k_{x}$ direction for the situation $\mathbf{L}, \mathbf{M}, \mathbf{N}$, which are denoted by red solid dots in panel a. (b)(e) for case-2 and (c)(f) for case-3.

properties of transport, we introduce another dimensionless parameter - residual ratio of AHC of the tilted WSM,

$$
R_{\mathrm{Hall}}=\frac{\sigma_{y z}}{\sigma_{0, y z}}=\frac{\int_{-k_{0}}^{k_{0}} \frac{d k_{x}}{2 \pi} \sigma_{y z, k_{x}}}{\sigma_{0, y z}}
$$

where $\sigma_{0, y z}$ is the AHC for type-I WSM and can be used to normalize the AHC in the tilting process. $\sigma_{y z, k_{x}}$ is the $\mathrm{AHC}$ of the $2 \mathrm{D}$ yz-slice for $k_{x} \in\left(-k_{0}, k_{0}\right)$.

Method: The method we use to calculate Hall conductivity is Landauer-Büttiker theory. The Hall conductivity is described by four-terminal formalism[36, 37],

$$
\sigma_{y z, k_{x}}=\frac{e^{2}}{h}\left(T_{12}-T_{14}\right)
$$

The transmission coefficient $T_{p q}\left(E_{F}\right)$ from the terminal $p$ to terminal $q$ is defined by

$$
T_{p q}\left(E_{F}\right)=\operatorname{Tr}\left[\Gamma^{p} G_{c}^{r} \Gamma^{q} G_{c}^{a}\right]
$$

where $p, q=1,2,3,4$, and they are ordered clockwise. Terminal-1 and terminal-3 (terminal-2 and terminal-4) are attached to the surface along the $+y(+z)$-direction acting as the measurement electrodes. The coupling matric $\Gamma^{p}$ is determined by the self-energy at the terminal

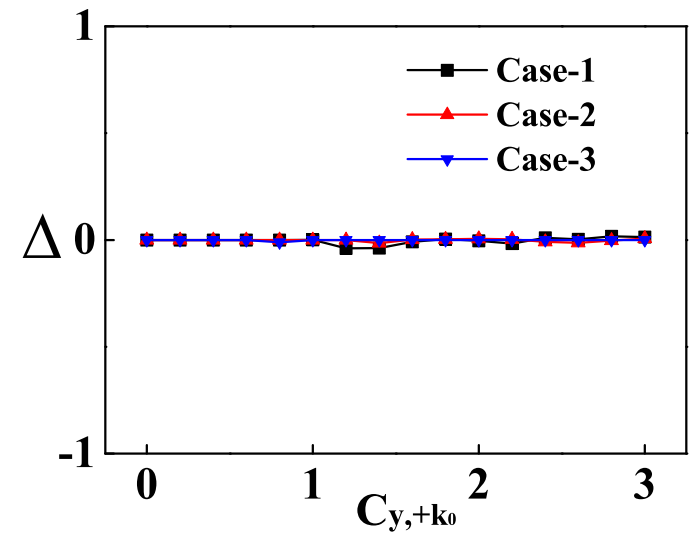

FIG. 6: (Coloronline) The intrinsic relationship between $R_{\mathrm{FA} 1}$ and $R_{\text {Hall }}$. Residual Hall conductivity is a physical consequence of residual class-1 Fermi arc.

$p, \Gamma^{p}=i\left[\sum^{p}-\left(\sum^{p}\right)^{\dagger}\right.$, the self-energy is acted as

$$
\sum^{p}=H_{p c}^{\dagger} g_{p}^{s} H_{p c}
$$

where $H_{p c}$ is the coupling matrix between terminal $p$ and center device area, $g_{p}^{s}$ is the surface Green's function of the lead $p . G_{c}^{r}\left(G_{c}^{a}\right)$ is the retarded (advanced) Green's function of center device area

$$
G_{c}^{r}\left(E_{F}+i \eta\right)=\left[E_{F}+i \eta-H_{c}-\sum_{p=1}^{4} \sum^{p}\right]^{-1}
$$

where $H_{c}$ is the real-space Hamiltonian of center device area, and $\eta$ is a infinite small value.

To obtain $T_{p q}$ efficiently, we simplify the calculation of $g_{p}^{s}$ and $G_{c}^{r}\left(G_{c}^{a}\right)$. On the one hand, in order to determine $g_{p}^{s}$ for these side-attached leads, the semi-infinite leads are sketched with discrete effective principal layers. These layers are defined as the smallest group of neighboring atomic planes and they allow only nearestneighbor interaction between them. So we effectively transforms the original system into a linear chain of principal layers [38-41]. Because the number of iterations required for convergence is smaller than any other method, this technique is valid $[42,43]$. On the other hand, to calculate $G_{c}^{r}$ for a large real-space system, it requires a full inversion of the 3D Hamiltonian. In fact, $G_{c}^{r}$ and $\Gamma^{p}$ is the function of $\sum^{p}$, and the matrix elements of $\sum^{p}$ are equal to 0 except for the elements corresponding to the surface of center device area which means $\sum^{p}$ is a large sparse matrix. So we can firstly figure out the Green's function $G_{c, \text { out }}^{r}$ and $\Gamma_{\text {out }}^{p}$ which only contain the elements related to outmost layer of the center device area. It is easy to deduce that

$$
T_{p q}=\operatorname{Tr}\left[\Gamma_{\text {out }}^{p} G_{c, \text { out }}^{r} \Gamma_{\text {out }}^{q} G_{c, \text { out }}^{a}\right],
$$

and the Green's function of the full device area can be calculated by Dyson equation $G=G^{0}+G^{0} V G$. 
As a result, the transform from 3D full real-space lattice to 2D out-most layer lattice dramatically improved the computational efficiency: the processing times for an initial 3D lattice is scaled as $O\left[\left(N_{x} N_{y} N_{z}\right)^{4}\right]$; For a 2D $y z$-slice is $O\left[N_{x}\left(N_{y} N_{z}\right)^{4}\right]$; For the outmost edge of the $y z$-slice is $O\left[N_{x} N_{y}\left(N_{z}\right)^{4}\right]$.

The result and generalization: From the results, we found that the residual ratio of AHC $R_{\text {Hall }}$ via tilting strength of nodes (shown in Fig.5) are almost the same as the residual ratio of FA1 $R_{\mathrm{FA} 1}$ via tilting strength of nodes (shown in Fig.2-4). Fig.5d ( or e, f) shows the distribution of $\sigma_{y z, k_{x}}$ along $k_{x}$-direction for the points $\mathbf{L}, \mathbf{M}, \mathbf{N}$, which are denoted by red solid dots in panel Fig.5a (or b, c) respectively. When the tilting strength is larger than the critical value, i.e., $\left|C_{y,+k_{0}}\right|>1$ or $\left|C_{y,-k_{0}}\right|>1, R_{\text {Hall }}$ decreases in the three cases. In case1 for $C_{y,+k_{0}}>1$, the region of $\sigma_{y z, k_{x}}=1$ doesn't change near the $k_{x}=-k_{0}$ and with the increasing of $C_{y,+k_{0}}$ the region of $\sigma_{y z, k_{x}}=1$ shrinks; In case-2 the region of $\sigma_{y z, k_{x}}=1$ pins at the middle of Fermi arc and the region of $\sigma_{y z, k_{x}}=1$ shrinks with the increasing of $C_{y,+k_{0}}$; In case-3, the region of $\sigma_{y z, k_{x}}=1$ is separated into two pieces near the two Weyl points and $R_{\text {Hall }}$ begins to decrease at $C_{y,+k_{0}} \simeq 0.7$ and rapidly turns into 0 before $C_{y,+k_{0}}=1$.

Finally we emphasize the intrinsic relationship between the residual ratio of FA1 $R_{\mathrm{FA} 1}$ and the residual ratio of AHC $R_{\text {Hall }}$, i.e.,

$$
R_{\text {Hall }} \simeq R_{\mathrm{FA} 1} .
$$

and the difference of the two dimensionless parameters $\Delta$ is figured in Fig.6. This result indicates that the residual Hall conductance comes from the contribution of the residual FA1.

It is known that the Hall conductivity is a physical consequence of Fermi arc and becomes a nontrivial topological property for type-I WSM. The intrinsic relationship between the residual ratio of FA1 and the residual ratio of AHC indicates that the residual Hall conductivity is a physical consequence of residual FA1 and also becomes a nontrivial topological property for hybrid and type-II WSM.

In general, a material may have multiple pairs of nodes and different nodes in the same system may belong to different types. For example, as the inversion symmetry- breaking material, the TaAs family belongs to type-I WSM which contains 24 bulk Weyl cones, and $\mathrm{WTe}_{2}$ belongs to type-II WSM which contains eight Weyl cones. And in the systems that don't have both inversion and time-reversal symmetries, one may find hybrid WSM with mixed types of Weyl nodes. Similar to the WSM with a pair of Weyl nodes, we may generalize our approach to those complicated systems and define several residual ratios of $\mathrm{FA} 1 / \mathrm{AHC}$ as

$$
\widetilde{R}_{\mathrm{FA} 1}=\sum_{i} \frac{\mathbf{d}_{\mathrm{FA} 1} \cdot \mathbf{e}_{k}}{d_{0, i}}, \quad \widetilde{R}_{\text {Hall }}=\sum_{i} \frac{\sigma_{i}}{\sigma_{0, i}},
$$

where we sum over the length of all pieces of Fermi arcs in the system along $\mathbf{e}_{k}$-direction in momentum space. Similarly, the intrinsic relationship between $\widetilde{R}_{\text {arc }}$ and $\widetilde{R}_{\text {Hall }}$ is similar to Eq.13.

\section{CONCLUSION AND DISCUSSION}

In conclusion, we find that there exist two classes of Fermi arcs in a Weyl semimetal. Based on a tight-binding model, the evolution and transport properties of the two classes of Fermi arcs are studied. The results show that the FA1 shrinks with the increasing of the tilting strength of the Weyl cones, which results to the decrease of anomalous Hall conductance. To characterize the interplay effect between the FA1 and AHC, we introduce two dimensionless parameters - the residual ratio of FA1 $R_{\mathrm{FA} 1}$ and the residual ratio of AHC $R_{\text {Hall }}$. In particular, there exists an intrinsic relationship between $R_{\mathrm{FA} 1}$ and $R_{\text {Hall }}$, i.e., $R_{\mathrm{FA} 1} \simeq R_{\text {Hall }}$. This result indicates that the residual Hall conductivity is a physical consequence of residual class-1 Fermi arc and that becomes a nontrivial topological property for hybrid or type-II Weyl semimetal. Therefore, this work provides an intuitive method to learn topological properties of Weyl semimetals.

\section{Acknowledgments}

This work is supported by NSFC under Grants No. $11474025,11504285,11674026$, SRFDP, and the Young Talent fund of the University Association for Science and Technology in Shaanxi, China.
[1] A. A. Burkov, and L. Balents, Phys. Rev. Lett. 107 127205 (2011).

[2] X. Wan, A. M. Turner, A. Vishwanath, and S. Y. Savrasov, Phys. Rev. B 83, 205101 (2011).

[3] H. B. Nielsen, and M. Ninomiya, Phys. Lett. B 130, 389 (1983).

[4] G. Xu, H. Weng, Z. Wang, X. Dai, and Z. Fang, Phys. Rev. Lett. 107, 186806 (2011).

[5] K. Y. Yang, Y. M. Lu, and Y. Ran, Phys. Rev. B, 84,
075129 (2011).

[6] P. Hosur, and X. L. Qi, C. R. Physique, 14, 857 (2013).

[7] J. Liu, and D. Vanderbilt, Phys. Rev. B, 90, 155316 (2014).

[8] M. Hirayama, R. Okugawa, S. Ishibashi, S. Murakami, and T. Miyake, Phys. Rev. Lett. 114206401 (2015).

[9] H. Weng, C. Fang, Z. Fang, B. A. Bernevig, and X. Dai, Phys. Rev. X 5, 011029 (2015).

[10] S. M. Huang, et al., Nat. Commun. 6, 7373 (2015). 
[11] L. Lu, Z. Wang, D. Ye, L. Ran, L. Fu, J. D. Joannopoulos, and M. Soljacic, Science 349, 622 (2015).

[12] S. Y. Xu, et al., Science 349, 613 (2015).

[13] B. Q. Lv, et al., Nat. Phys. 11, 724 (2015).

[14] C. Shekhar, et al., Nat. Phys. 11, 645 (2015).

[15] S. Y. Xu, et al., Nat. Phys. 11, 748 (2015).

[16] H. Nielsen, and N. Ninomiya, Phys. Lett. B 130, 389 (1983).

[17] V. Aji, Phys. Rev. B 85, 241101(R) (2012).

[18] T. Son, and N. Yamamoto, Phys. Rev. Lett. 109, 181602 (2012).

[19] A. A. Zyuzin, and A. A. Burkov, Phys. Rev. B 86, 115133 (2012).

[20] B. Q. Lv, et al., Phys. Rev. X 5, 031013 (2015).

[21] L. X. Yang, et al., NaturePhys. 11, 728 (2015).

[22] D. T. Son, and B. Z. Spivak, Phys. Rev. B 88, 104412 (2013).

[23] X. Huang, et al., Phys. Rev. X 5, 031023 (2015).

[24] S. K. Jian , Y. F. Jiang, and H. Yao, Phys. Rev. Lett., 114, 237001 (2015).

[25] A. A. Soluyanov, et al., Nature 527495 (2015).

[26] Z. Wang, et al., Phys. Rev. Lett. 117, 056805 (2016).

[27] S. Kourtis, J. Li, Z. Wang, A. Yazdani, and B. A. Bernevig, Phys. Rev. B 93, 041109 (2016).

[28] Y. Sun, S. C. Wu, M. N. Ali, C. Felser, and B. Yan, Phys. Rev. B 92161107 (2015).

[29] Y. Qi, P. G. Naumov, et al. Nat. Commun. 711038
(2016)

[30] T. R. Chang, S. Y. Xu, G. Chang, et al. Nat. Commun. 710639 (2016).

[31] X. Kong, et al., Phys. Rev. A.95, 033629 (2017).

[32] F.Y. Li, X. Luo, X. Dai, Y. Yu, F. Zhang and G. Chen, Phys. Rev. B 94, 121105 (2016)

[33] B. I. Halperin, Phys. Rev. B 25, 2185 (1982).

[34] A. H. MacDonald and P. Streda, Phys. Rev. B 29, 1616 (1984).

[35] A. A. Zyuzin, and R. P. Tiwari, JETP letters 103, 717 (2016).

[36] M. Buttiker, Phys. Rev. Lett. 57, 1761 (1986).

[37] J. Li, L. Hu, and S. Q. Shen, Phys. Rev. B 71, 241305(R) (2005).

[38] M. P. Lpez Sancho, J. M. Lpez Sancho, and J. Rubio, J. Phys. F: Met 14, 1205 (1984).

[39] P. Dutta, S. K. Maiti, and S. N. Karmakar, J. Appl. Phys. 114, 034306 (2013).

[40] V. Meunier, and B. G. Sumpter, J. Chem.Phys. 123, 24705 (2005).

[41] E. Jódar, A. Pérez-Garrido, and A. Díaz-Sánchez, Phys. Rev. B 73, 205403 (2006).

[42] T. C. Li, and S. P. Lu, Phys. Rev. B 77, 085408 (2008).

[43] F. Khoeini, A. A. Shokri, and F. Khoeini, Eur. Phys. J. B 75, 505 (2010). 